hep-ph/9610249

Revised

\title{
On the extreme behaviour of $g_{1}\left(x, Q^{2}\right)$ at $x \rightarrow 0$.
}

\author{
S. M. Troshin \\ Institute for High Energy Physics, \\ Protvino, Moscow Region, 142284 Russia
}

\begin{abstract}
On the basis of the $U$-matrix form of $s$-channel unitarization, we consider constraints unitarity provides for the spin structure function $g_{1}\left(x, Q^{2}\right)$ at $x \rightarrow 0$. Corresponding constraint for the spin structure function $h_{1}\left(x, Q^{2}\right)$ is given along.
\end{abstract}

PACS: $11.80 . \mathrm{Fv}, 13.60 . \mathrm{Hb}, 13.88 .+\mathrm{e}$ spin structure functions, unitarity, low- $x$ 
The problem of spin structure of a nucleon during the last decade has become a very significant one. Experiments in this field continue to bring new facts which can change earlier interpretations. As it appears now, the behaviour of the function $g_{1}$ at $x \rightarrow 0$ is becoming crucial in the evaluation of the total hadron helicity carried by quarks. Recent experimental results indicate that the function $g_{1}(x)$ might have a rising behaviour at $x \rightarrow 0$ [1] contrasting to a smooth Regge dependence $g_{1} \sim x^{-\alpha_{a_{1}}}$ (with $-0.5<\alpha_{a_{1}}<0$ ) used in the experimental analysis. Indeed, the dependence $g_{1}^{n}(x)=-0.2 / x^{0.8}$ observed at small $x$ in the experiment E154 at SLAC [1] when taken as an extrapolation to $x=0$ could significantly change the first moment of the structure function $g_{1}$. Thus, the problem of small- $x$ seems to be important in both cases of unpolarized and polarized DIS.

It has been discussed in several papers basing on some general considerations [2], DLA in QCD [3] and on the model approaches [4, 6, 6]. Various forms have been discussed including the extreme one $x g_{1} \sim \log ^{2} x$. In this note we consider bound for $g_{1}$ by taking account of unitarity in the direct channel. Corresponding constraint for the transversity $h_{1}$ is given also.

For that purpose it is convinient to use the relation between the distribution functions and the discontinuites in the helicity amplitudes of the forward antiquark-hadron scattering [7] which is based on the dominance of the "handbag" diagrams in DIS:

$$
\begin{aligned}
q(x) & =\left.\frac{1}{2} \operatorname{Im}\left[F_{1}(s, t)+F_{3}(s, t)\right]\right|_{t=0} \\
\Delta q(x) & =\left.\frac{1}{2} \operatorname{Im}\left[F_{3}(s, t)-F_{1}(s, t)\right]\right|_{t=0},
\end{aligned}
$$

where $s \simeq Q^{2} / x$ and $F_{i}$ are the helicity amplitudes for the elastic quark-hadron scattering in the notations for the nucleon-nucleon scattering, i.e.

$$
F_{1} \equiv F_{1 / 2,1 / 2,1 / 2,1 / 2}, F_{2} \equiv F_{1 / 2,1 / 2,-1 / 2,-1 / 2}, F_{3} \equiv F_{1 / 2,-1 / 2,1 / 2,-1 / 2}, F_{4} \equiv F_{1 / 2,-1 / 2,-1 / 2,1 / 2}
$$

and

$$
F_{5} \equiv F_{1 / 2,1 / 2,1 / 2,-1 / 2} .
$$

We consider a quark-hadron scattering similar to a hadron-hadron scattering. It can be justified when considering quark as a structured hadronlike object. Arguments in favour of such approach can be found in [8, 9]. We do not discuss here a possible influence of the off-mass-shell effects.

Unitary representation for helicity amplitudes in the $U$-matrix form of unitarization provides the following relations [10] in the impact parameter representation:

$$
F_{\Lambda_{1}, \lambda_{1}, \Lambda_{2}, \lambda_{2}}(s, b)=U_{\Lambda_{1}, \lambda_{1}, \Lambda_{2}, \lambda_{2}}(s, b)+i \rho(s) \sum_{\mu, \nu} U_{\Lambda_{1}, \lambda_{1}, \mu, \nu}(s, b) F_{\mu, \nu, \Lambda_{2}, \lambda_{2}}(s, b),
$$

where $\lambda_{i}$ and $\Lambda_{i}$ are the quark and hadron helicities, respectively, and $b$ is the impact parameter. The kinematical function $\rho(s) \simeq 1$ at $s \gg 4 m^{2}$. Explicit solution of Eqs. (2) has a rather complicated form:

$$
F_{1}(s, b)=\frac{\tilde{U}_{1}(s, b)\left[1-i U_{1}(s, b)\right]-i \tilde{U}_{2}(s, b) U_{2}(s, b)}{\left[1-i U_{1}(s, b)\right]^{2}-\left[U_{2}(s, b)\right]^{2}},
$$




$$
F_{3}(s, b)=\frac{\tilde{U}_{3}(s, b)\left[1-i U_{3}(s, b)\right]-i \tilde{U}_{4}(s, b) U_{3}(s, b)}{\left[1-i U_{3}(s, b)\right]^{2}-\left[U_{4}(s, b)\right]^{2}}
$$

where

$$
\tilde{U}_{i}(s, b)=U_{i}(s, b)+2 U_{5}(s, b) F_{5}(s, b)
$$

and

$$
F_{5}(s, b)=\frac{U_{5}(s, b)}{\left[1-i U_{1}(s, b)-i U_{2}(s, b)\right]\left[1-i U_{3}(s, b)-i U_{4}(s, b)\right]-4 U_{5}^{2}(s, b)} .
$$

However, in the approximation when the helicity-flip functions are much less than the helicity nonflip ones one can get simple expressions

$$
F_{1,3}(s, b)=\frac{U_{1,3}(s, b)}{1-i U_{1,3}(s, b)}
$$

The functions $F_{i}(s, t)$ are the corresponding Fourier-Bessel transforms of the functions $F_{i}(s, b)$ :

$$
F_{1,3}(s, t)=\frac{s}{\pi^{2}} \int_{0}^{\infty} b d b F_{1,3}(s, b) J_{0}(b \sqrt{-t}) .
$$

Unitarity requires that $\operatorname{Im} U_{1,3}(s, b) \geq 0$. Assume for simplicity that the functions $U_{1,3}(s, b)$ are pure imaginary, i.e. $U_{1,3}(s, b) \rightarrow i U_{1,3}(s, b)$, and parametrice these functions in the form

$$
\begin{aligned}
U_{1}(s, b) & =\frac{1}{2}(1-a) U(s, b) \\
U_{3}(s, b) & =\frac{1}{2}(1+a) U(s, b),
\end{aligned}
$$

where the function $U(s, b)$ correspond to the unpolarized case and $|a| \leq 1$. To maximaze the difference $U_{3}(s, b)-U_{1}(s, b)=a U(s, b)$ we consider $a$ as a constant. For the function $U(s, b)$ we use simple form

$$
U(s, b)=g s^{\lambda} e^{-\mu b} .
$$

This is a rather general parameterization for $U(s, b)$ which provides correct analytical properties in the complex $t$-plane. It follows also, for example, from the chiral quark model for $U$-matrix [11]. Note, that the following spectral representation is valid for the function $U(s, b)$ [12]:

$$
U(s, b)=\frac{\pi^{2}}{s} \int_{t_{0}}^{\infty} \rho(s, t) K_{0}(b \sqrt{t}) d t .
$$

Another form of $U(s, b)$, e.g

$$
U(s, b)=g s^{\lambda} e^{-b^{2} / \omega(s)}, \quad \omega(s) \sim \log s,
$$

which also leads to the total cross-section saturating the Froissart-Martin bound would provide the same results, however, it does not respect analytical properties in the complex $t$ plane. Indeed, as it follows from Eq. (8) the function $U(s, b)$ should have a linear exponential dependence on the impact parameter at large $b$. 
Then using Eq. (17) as an explicit form for $U(s, b)$ in Eqs. (3) and (4) it can be shown that Eqs. (1) provide at $x \rightarrow 0$

$$
\begin{aligned}
& \Delta q(x) \sim a /\left(1-a^{2}\right) \log x / x \text { for }|a| \neq 1 \\
& \Delta q(x) \sim \log ^{2} x / x \text { for }|a|=1
\end{aligned}
$$

and

$$
q(x) \sim \log ^{2} x / x .
$$

Since the above result are valid for each quark flavour the similar behaviour at $x \rightarrow 0$ will take place for the function $g_{1}(x)$. Thus, unitarity together with parameterization of the $U_{-}$ matrix which provides saturation of the Froissart-Martin bound lead to the following upper bounds for the structure function $g_{1}(x)$ :

$$
g_{1}(x) \leq \log x / x \quad(|a| \neq 1)
$$

and

$$
g_{1}(x) \leq \log ^{2} x / x \quad(|a|=1) .
$$

The latter bound has been obtained earlier in [2].

Using the relation between the function $h_{1}^{q}(x)$ and corresponding quark-hadron helicity amplitude $F_{2}(s, t)[7]$ :

$$
h_{1}^{q}(x)=\left.\frac{1}{2} \operatorname{Im} F_{2}(s, t)\right|_{t=0}
$$

we can get similar upper bound for $h_{1}(x)$ at small $x$. This function measures the number density of quarks in the transversity eigenstates and is determined as a matrix element of the twist-two transversity operator.

The unitary representation for the function $F_{2}(s, t)$ has the following form [10]:

$$
F_{2}(s, t)=\frac{s}{\pi^{2}} \int_{0}^{\infty} b d b \frac{U_{2}(s, b)}{\left[1-i U_{1}(s, b)\right]^{2}} J_{0}(b \sqrt{-t}) .
$$

Using Eqs. (13), (14) and proceeding through the same steps as in the case of $g_{1}(x)$ we arrive to the following extreme behaviour of $h_{1}(x)$ at $x \rightarrow 0$ :

$$
h_{1}(x) \sim \log x / x
$$

Such behaviour of $h_{1}(x)$ could be considered, in particular, as an indirect confirmation of the inequality obtained in [7].

To have such upper bounds seems to be useful nowadays when the experimental data indicate the possible rising behaviour of $g_{1}(x)$ at small $x$ and the studies of $h_{1}(x)$ are planned at RHIC.

The author is grateful to N. E. Tyurin for his helpful comments and discussions. 


\section{References}

[1] G. Gates (E154 Collaboration), Talk given at 12th International Symposium on High-Energy Spin Physics, Amsterdam, September 10-14, 1996.

[2] F. E. Close and R. G. Roberts, Phys. Lett. B336 (1994) 257.

[3] J. Bartels, B. I. Ermolaev and M. G. Ryskin, Z. Phys. C70 (1996) 273;

J. Blümlein and A. Vogt, Preprint DESY 96-050.

[4] S. D. Bass and P. V. Landshoff, Phys. Lett. B336 (1994) 537.

[5] A. E. Dorokhov, N. I. Kochelev and Yu. A. Zubov, Int. J. Mod. Phys. A8 (1993) 603.

[6] S. V. Goloskokov, hep-ph/9604261.

[7] R. L. Jaffe and X. Ji, Nucl. Phys. B375 (1992) 527;

J. Soffer, Phys. Rev. Lett. 74 (1995) 1292.

[8] V. Del Duca, S. J. Brodsky and P. Hoyer, Phys. Rev. D46 (1992) 931.

[9] J. D. Bjorken, SLAC-PUB-95-6949; SLAC-PUB-7096, 1996.

[10] V. F. Edneral, S. M. Troshin and N. E. Tyurin, JETP Lett. 30 (1979) 330.

[11] S. M. Troshin and N. E. Tyurin, Nuovo Cim. 106A (1993) 327; Phys. Rev. D49 (1994) 4427.

[12] S. M. Troshin and N. E. Tyurin, Theor. Math. Phys. 50 (1982) 150. 